\title{
VARIABILITÉ SELON LE CAPTUREUR DE L'EFFICACITÉ DE RÉPULSIFS À BASE D'HUILES VÉGÉTALES DANS LA PROTECTION INDIVIDUELLE CONTRE LES PIQÛRES DE SIMULIUM DAMNOSUM S. L.
}

\author{
SYLLA M.*, KONAN L.**, DOANNIO J.M.C.** \& TRAORE S.**
}

Summary : EFFECT OF INDIVIDUAL ATTRACTION ON THE EFFICIENCY OF VEGETAL OILS AGAINST SIMULIUM DAMNOSUM S. L. BITES

In Ivory Coast, treatments against black flies larva are interrupted since December 1998 and these flies are back everywhere in abundance. They are now a severe source of discomfort, so it is therefore very important to propose to rural populations a personal protection method against their bites. This article is about the individual attraction of fly boys on the efficiency of lotions and creams of the different repellents (coconut, palm nut, gobi). Our works were carried out in savanna zone (Niakaramandougou) and in forest zone (Soubré) in Ivory Coast. The methodology consisted in capturing black flies on volunteers whose legs were rubbed with repellent. Coconut, palm nut and gobi formulas (lotions and creams) were tested on Simulium damnosum s. I During each session, which lasted from 07:00 am to 12:00 and from 03:00 pm to 06:00 pm, one person without repellent was used as a control person. The results show that the least efficient repellent (gobil ensures nonetheless a reduction of $90 \%$ of the number of bites that an individual would have received if he was not protected by the repellent. Experimentations allows to say that for a given repellent and formulas (lotion and cream), there is no significant difference between the number of Simulium damnosum s. I. bites received by fly boys

KEY WORDS : Simulium damnosum s. I., nuisance, repellent, individual attraction.

\section{Résumé :}

Dans le présent article, nous faisons état de la variabilité selon le captureur de l'efficacité de répulsifs à base d'huiles végétales appliquées sur la peau contre les piqûres de Simulium damnosum s. I. Les expérimentations ont été effectuées en zones de savane (Chaussée-Niaka) et de forêt (Soubré) de Côte d'lvoire. Les formulations (lotions, crèmes) testées lors de ces travaux ont été obtenues à partir d'huiles d'amande de palme (Elaeis guineensis), de coco (Cocos nucifera) et de gobi (Carapa procera). La technique classique de capture sur homme à l'aide de tubes à hémolyse en matière plastique a été utilisée. Pour chaque journée d'expérimentation, un captureur non traité ljambes non enduites de répulsiff servait de témoin. Les résultats indiquent qu'en savane, quels que soient le répulsif et le captureur, la densité simulidienne se situait de 1 à 4,1 à 6 et de 2 à 10 simulies par homme et par jour lorsque le captureur était respectivement protégé avec le coco, le palmiste et le gobi. Avec les mêmes répulsifs, en zone de forêt, l'agressivité simulidienne variait de 9 à 16, de 2 à 30 et de 8 à 49 piqûres par jour. En comparaison, le témoin recevait de 67 à 90 et de 310 à 533 piqûres par jour, respectivement en zones de savane et de forêt. Malgré les variations importantes dans le nombre de simulies capturées en fonction du captureur choisi, nos résultats montrent que quels que soient le répulsif et l'espèce simulidienne, une protection similaire est conférée à tous les captureurs. Ils indiquent également que le recours à des substances naturelles locales, utilisées comme répulsifs en application dermique, est l'une des meilleures méthodes de protection personnelle contre les simulies.

MOTS CLÉS : Simulium damnosum s. I., nuisance, répulsifs, attractivité individuelle.

à l'exploitation des terres dans d'autres zones, si le seuil de tolérance qui est de 30 piqûres par homme et par jour est largement dépassé (Hougard et al., 1998). L'expérience d'OCP indique que le problème de la nuisance simulidienne pourrait être résolu au niveau des sites d'intérêt socio-économique par le traitement au sol des gites à l'aide de larvicides financés par les complexes agro-industriels. Par contre, pour les communautés dépourvues de moyens financiers, la seule solution réside dans la protection individuelle (ou collective) contre les piqûres de simulies par l'usage de produits répulsifs ou le port de vêtements protecteurs. En Afrique, les travaux de Lewis (1953) au Soudan, Schmidt (1977) en Éthiopie, Renz et Enyong (1987) au Cameroun, Noamesi et al. (1970) puis Opuku et al. (1985) au Ghana, et Adossi (1984) en Côte d'Ivoire, ont démontré l'efficacité de nombreux répulsifs à base

* Centre d'entomologie médicale et vétérinaire, 01 BP 2597, Bouaké 01, Côte d'Ivoire.

*** Centre Pierre Richet, 01 BP 1500 Bouaké, 01, Côte d'Ivoire. Correspondance : Traoré Soungalo.

Tél. : (225) 31633746 - E-mail : pefoungo@yahoo.fr 
de pyréthrinoïdes contre Simulium damnosum s. 1. Néanmoins leur utilisation n'est pas courante car du fait de leurs coûts (Hougard et al., 1998) les populations rurales délaissent ces répulsifs vendus en officine, pour se tourner vers les recettes traditionnelles.

Au terme des expérimentations menées en zone de forêt et de savane de Côte d'Ivoire, Pitroipa (2000) a conclut que l'huile d'amandes de palme (Elaeis guineensis) et l'huile de coco (Cocos nucefera) présentaient de réelles capacités répulsives contre les piqûres de Simulium damnosum s. 1. En effet, elles maintiennent en permanence le nombre de piqûres par homme et par jour en dessous du seuil de tolérance, d'où l'absence de nuisance pour la personne protégée par ces répulsifs. Toutefois, dans ses conclusions, il a souligné la nécessité de tester plusieurs formulations d'un même produit, avant d'aboutir à celle qui procure la meilleure protection.

Dans le présent article, nous faisons état de la variabilité selon le captureur de l'efficacité de lotions et de crèmes de répulsifs à base d'huiles végétales contre les piqûres de Simulium damnosum s. 1.

\section{MATÉRIEL ET MÉTHODES}

\section{ZONES D'ÉTUDE}

I a première expérimentation a été effectuée à Chaussée-Niaka, en zone de savane, dans la sous$\triangle$ préfecture de Niakaramandougou ( $8^{\circ} 40^{\prime}$ latitude nord- $5^{\circ} 17^{\prime}$ longitude ouest). La seconde expérimentation a été réalisée en forêt, dans le département de Soubré $\left(5^{\circ} 37^{\prime}\right.$ à $6^{\circ} 40^{\prime}$ 'de latitude nord- $5^{\circ} 47^{\prime}$ à $6^{\circ} 37^{\prime}$ de longitude ouest).

\section{MATÉRIEL DE LA CAPTURE SUR HOMME}

L'équipement de la capture sur homme est classique (une montre, des tubes à hémolyse, du coton hydrophile, des étiquettes, des bracelets élastiques et/ou un petit sac en toile) et son utilisation a été décrite par plusieurs auteurs (Le Berre, 1966; Duke, 1968; Philippon, 1977)

\section{RÉPULSIFS À BASE D'HUILES VÉGÉTALES}

Les formulations (lotions, crèmes) testées lors de nos expérimentations ont été obtenues à partir d'huiles végétales. Les méthodes d'extraction des huiles d'amande de palme (Elaeis guineensis), de coco (Cocos nucifera) et de gobi (Carapa procera) ont déjà été décrites (Pitroipa, 2000; Sylla, 2001).

Les formulations dénommées "lotions" ont été obtenues en mélangeant de l'huile brute végétale à base de coco, palmiste ou gobi et un support (beurre de karité ou vaseline) dans les proportions respectives de $75 \%$ et
$25 \%$. Dans les formulations "crèmes", par contre, les proportions d'huile végétale et du support sont de $50 \%$ pour chaque produit.

\section{APPLICATION DES RÉPULSIFS}

Au début de chaque journée de capture, les captureurs sélectionnés se lavent les jambes et les bras avec du savon non parfumé, dénommé savon de Marseille. Le répulsif (lotion ou crème) est appliqué à raison d'un gramme par jambe, soit deux grammes par captureur. Le produit est mesuré avec une petite cuillère dont le contenu équivaut à un gramme. Il est déposé dans le creux de la main du captureur qui l'étale entre ses deux paumes puis le frotte sur toute la surface de la jambe (du genou au pied).

Au total, douze répulsifs (six "lotions" et six "crèmes") ont été testés en zone de savane, puis en zone de forêt. Pour une journée de capture donnée, chaque répulsif a été appliqué deux fois (à 7 heures et à 15 heures).

\section{CAPTURE DES FEMELLES PIQUEUSES DE SIMULIUM DAMNOSUM S. L.}

La technique classique de capture sur homme à l'aide de tubes à hémolyse en matière plastique a été utilisée (Philippon, 1977). Quatre points de capture ont été retenus sur le site de savane et autant sur celui de forêt.

Huit volontaires, à raison de quatre par zone, ont été choisis pour participer à l'étude. Ils sont de sexe masculin et leur âge moyen était de 25 ans. Sur chaque site, les quatre captureurs ont été installés individuellement aux points de capture retenus. Pour chaque journée d'expérimentation, un des captureurs, non enduit de répulsif, servait de témoin. Par ailleurs, pour chaque point de capture donné, chaque répulsif a été testé deux fois. Il est à noter qu'un système de rotation a permis à chaque captureur (témoin y compris) de visiter tous les points de capture. Les captures journalières s'étalaient de 7 heures à 12 heures, puis de 15 heures à 18 heures. Toutes les heures, les simulies capturées étaient comptabilisées et les tubes regroupés en un lot étiqueté portant la date, l'heure et le nom du captureur.

\section{ANALYSE DES RÉCOLTES}

Au terme de chaque séance de capture, les simulies sont acheminées au laboratoire où elles sont d'abord tuées au chloroforme, puis identifiés d'après les caractères micromorphologiques (Kurtak et al., 1981; Garms et Zillmann, 1984; Wilson et al., 1993). Pour chaque répulsif, nous avons déterminé le pourcentage de protection totale par captureur pour la durée de l'expériementation (Schreck et al., 1979). Puis les différents pourcentages ont été comparés à l'aide du test Chi deux. 


\section{RÉSULTATS}

L e tableau I indique qu'en savane, quels que soient le répulsif et le captureur, les densités simulidiennes dse situait de 1 à 4,1 à 6 et de 2 à 10 simulies par homme et par jour lorsque le captureur était respectivement protégé avec le coco, le palmiste et le gobi. Avec les mêmes répulsifs, en zone de forêt (tableau III), l'agressivité simulidienne variait de 9 à 16 , de 2 à 30 et de 8 à 49 piqûres par jour. En comparaison, le témoin recevait de 67 à 90, et de 310 à 533 piqûres par jour, respectivement en zones de savane et de forêt.

En zone de savane, les pourcentages de réduction du nombre de piqûres par rapport au témoin variaient de 93,4 à $98,7 \%, 91,5$ à $97,9 \%$, et de 88,6 à $98,1 \%$ respectivement avec le coco, le palmiste et le gobi. Pour ces mêmes répulsifs, en zone de forêt, les pourcentages variaient de 96,1 à $97,7 \%, 94,4$ à 99,5\%, et de 90,7 à 97,4 \%

La comparaison deux à deux de l'efficacité des répulsifs selon le captureur, figure dans les tableaux II et IV. Au regard de ces tableaux, 23 des 36 tests statistiques indiquent une différence significative d'efficacité d'un même répulsif selon le captureur.
L'identification morphologique des simulies indique que celles de Chaussée-Niaka appartenaient essentiellement aux espèces savanicoles (sous complexe $S$. damnosum) et celles de Soubré aux espèces forestières (sous complexe S. sanctipauli).

\section{DISCUSSION}

$\mathrm{L}$ 'emploi de l'homme comme appât est le procédé le plus courant et le plus efficace pour la capture des simulies (Le Berre, 1966; Duke, 1968; Quillevere, 1979). Toutefois, cette méthode présente de nombreuses imperfections dont des variations importantes dans le nombre de simulies capturées en fonction du captureur choisi et des lieux de capture (Philippon, 1977). Malgré cette variabilité individuelle, les résultats obtenus par Pitroipa (2000) avec l'huile brute de coco indiquent une bonne concordance des taux de protection obtenus chez les captureurs. En effet, quel que soit le captureur, le pourcentage de réduction du nombre de piqûres par rapport au témoin est au moins égal à $99 \%$. Ce pourcentage se traduit par

\begin{tabular}{|c|c|c|c|c|c|}
\hline & & Captureur 1 & Captureur 2 & Captureur 3 & Captureur 4 \\
\hline \multirow[t]{3}{*}{ Témoin } & Nombre de jour de capture & 8 & 8 & 8 & 8 \\
\hline & Nombre de femelles capturées & 716 & 602 & 536 & 648 \\
\hline & Nombre de piqûres/homme/jour & 90 & 76 & 67 & 81 \\
\hline \multirow[t]{4}{*}{ Coco } & Nombre de jour de capture & 8 & 8 & 8 & 8 \\
\hline & Nombre de femelles capturées & 27 & 21 & 35 & 8 \\
\hline & Nombre de piqûres/homme/jour & 3 & 3 & 4 & 1 \\
\hline & Taux de protection & $96,22 \%$ & $96,51 \%$ & $93,47 \%$ & $98,76 \%$ \\
\hline \multirow[t]{4}{*}{ Palmiste } & Nombre de jour de capture & 8 & 8 & 8 & 8 \\
\hline & Nombre de femelles capturées & 34 & 51 & 11 & 33 \\
\hline & Nombre de piqûres/homme/jour & 4 & 6 & 1 & 4 \\
\hline & Taux de protection & $95,25 \%$ & $91,52 \%$ & $97,94 \%$ & $94,90 \%$ \\
\hline \multirow[t]{4}{*}{ Gobi } & Nombre de jour de capture & 8 & 8 & 8 & 8 \\
\hline & Nombre de femelles capturées & 81 & 68 & 16 & 12 \\
\hline & Nombre de piqûres/homme/jour & 10 & 9 & 2 & 2 \\
\hline & Taux de protection & $88,68 \%$ & $88,70 \%$ & $97,01 \%$ & $98,14 \%$ \\
\hline
\end{tabular}

Tableau I. - Variabilité de l'efficacité des formulations (lotions, crèmes) à base d'huiles de coco, de palmiste et de gobi selon le captureur en zone de savane.

\begin{tabular}{lcccccc}
\hline & $\mathbf{C}_{\mathbf{1}} \mathbf{x} \mathbf{C}_{\mathbf{2}}$ & $\mathbf{C}_{\mathbf{1}} \mathbf{x} \mathbf{C}_{\mathbf{3}}$ & $\mathbf{C}_{\mathbf{1}} \mathbf{x} \mathbf{C}_{\mathbf{4}}$ & $\mathbf{C}_{\mathbf{2}} \mathbf{x} \mathbf{C}_{\mathbf{3}}$ & $\mathbf{C}_{\mathbf{2}} \mathbf{x} \mathbf{C}_{\mathbf{4}}$ & $\mathbf{C}_{\mathbf{3}} \mathbf{x} \mathbf{C}_{\mathbf{4}}$ \\
\hline Coco & $\mathrm{p}=0,900$ & $\mathrm{p}=0,036$ & $\mathrm{p}=0,005$ & $\mathrm{p}=0,025$ & $\mathrm{p}=0,014$ & $\mathrm{~s}=2,710^{-6}$ \\
& $\mathrm{NS}$ & $\mathrm{S}$ & $\mathrm{S}$ & $\mathrm{S}$ & $\mathrm{S}$ \\
Palmiste & $\mathrm{p}=0,006$ & $\mathrm{p}=0,022$ & $\mathrm{p}=0,772$ & $\mathrm{p}=3,610^{-6}$ & $\mathrm{p}=0,023$ & $\mathrm{p}=0,009$ \\
& $\mathrm{~S}$ & $\mathrm{~S}$ & $\mathrm{NS}$ & $\mathrm{S}$ & $\mathrm{S}$ & $\mathrm{p}$ \\
Gobi & $\mathrm{p}=0,154$ & $\mathrm{p}<10^{-6}$ & $\mathrm{p}<10^{-6}$ & $\mathrm{p}<10^{-6}$ & $\mathrm{p}<10^{-6}$ & $\mathrm{p}=0,277$ \\
& $\mathrm{NS}$ & $\mathrm{S}$ & $\mathrm{S}$ & $\mathrm{S}$ & $\mathrm{S}$ \\
\hline
\end{tabular}

$\mathrm{C}_{1} \times \mathrm{C}_{2}=$ captureur 1 comparé au captureur 2 .

$\mathrm{NS}=$ non significatif $; \mathrm{S}=$ significatif.

Tableau II. - Comparaison deux à deux de l'efficacité des formulations (lotions, crèmes) à base d'huiles de coco, de palmiste et de gobi selon le captureur en zone de savane. 


\begin{tabular}{|c|c|c|c|c|c|}
\hline & & Captureur 1 & Captureur 2 & Captureur 3 & Captureur 4 \\
\hline \multirow[t]{3}{*}{ Témoin } & Nombre de jour de capture & 4 & 4 & 4 & 4 \\
\hline & Nombre de femelles capturées & 2131 & 1545 & 1471 & 1241 \\
\hline & Nombre de piqûres/homme/jour & 533 & 386 & 368 & 310 \\
\hline \multirow[t]{4}{*}{$\mathrm{Coco}$} & Nombre de jour de capture & 4 & 4 & 4 & 4 \\
\hline & Nombre de femelles capturées & 62 & 35 & 58 & 38 \\
\hline & Nombre de piqûres/homme/jour & 16 & 9 & 15 & 10 \\
\hline & Taux de protection & $97,09 \%$ & $97,73 \%$ & $96,05 \%$ & $96,93 \%$ \\
\hline \multirow[t]{4}{*}{ Palmiste } & Nombre de jour de capture & 4 & 4 & 4 & 4 \\
\hline & Nombre de femelles capturées & 119 & 45 & 6 & 23 \\
\hline & Nombre de piqûres/homme/jour & 30 & 11 & 2 & 6 \\
\hline & Taux de protection & $94,41 \%$ & $97,08 \%$ & $99,59 \%$ & $98,14 \%$ \\
\hline \multirow[t]{4}{*}{ Gobi } & Nombre de jour de capture & 4 & 4 & 4 & 4 \\
\hline & Nombre de femelles capturées & 197 & 44 & 38 & 32 \\
\hline & Nombre de piqûres/homme/jour & 49 & 11 & 10 & 8 \\
\hline & Taux de protection & $90,75 \%$ & $97,15 \%$ & $97,41 \%$ & $97,42 \%$ \\
\hline
\end{tabular}

Tableau III. - Variabilité de l'efficacité des formulations (lotions, crèmes) à base d'huiles de coco, de palmiste et de gobi selon le captureur en zone de forêt.

\begin{tabular}{|c|c|c|c|c|c|c|}
\hline & $\mathrm{C}_{1} \times \mathrm{C}_{2}$ & $\mathrm{C}_{1} \times \mathrm{C}_{3}$ & $\mathrm{C}_{1} \times \mathrm{C}_{4}$ & $\mathrm{C}_{2} \times \mathrm{C}_{3}$ & $\mathrm{C}_{2} \times \mathrm{C}_{4}$ & $\mathrm{C}_{3} \times \mathrm{C}_{4}$ \\
\hline Coco & $\begin{array}{c}\mathrm{p}=0,272 \\
\mathrm{NS}\end{array}$ & $\begin{array}{c}\mathrm{p}=0,108 \\
\mathrm{NS}\end{array}$ & $\begin{array}{c}\mathrm{p}=0,883 \\
\mathrm{NS}\end{array}$ & $\begin{array}{c}\mathrm{p}= \\
\mathrm{S}\end{array}$ & $\begin{array}{c}\mathrm{p}=0,234 \\
\mathrm{NS}\end{array}$ & $\begin{array}{c}\mathrm{p}=0,257 \\
\mathrm{NS}\end{array}$ \\
\hline Palmiste & $\begin{array}{c}\mathrm{p}=0,0001 \\
\mathrm{~S}\end{array}$ & $\begin{array}{c}\mathrm{p}<10^{-6} \\
\mathrm{~S}\end{array}$ & 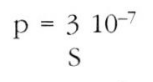 & $\begin{array}{c}\mathrm{p}=\mathrm{S}_{\mathrm{S}} \quad 10^{-7} \\
\end{array}$ & $\begin{array}{c}\mathrm{p}=0,093 \\
\mathrm{NS}\end{array}$ & $\mathrm{p}=\frac{5,4}{\mathrm{~S}} 10^{-4}$ \\
\hline Gobi & $\begin{array}{c}\mathrm{p}<0,10^{-6} \\
\mathrm{~S}\end{array}$ & $\begin{array}{c}\mathrm{p}<10^{-6} \\
\mathrm{~S}\end{array}$ & $\begin{array}{c}\mathrm{p}<10^{-6} \\
\mathrm{~S}\end{array}$ & $\begin{array}{c}p=0,737 \\
N S\end{array}$ & $\begin{array}{c}\mathrm{p}=0,751 \\
\mathrm{NS}\end{array}$ & $\begin{array}{c}\mathrm{p}=0,909 \\
\mathrm{NS}\end{array}$ \\
\hline
\end{tabular}

$\mathrm{C}_{1} \times \mathrm{C}_{2}=$ captureur 1 comparé au captureur 2 .

NS $=$ non significatif $; S=$ significatif.

Tableau IV. - Comparaison deux à deux de l'efficacité des formulations (lotions, crèmes) à base d'huiles de coco, de palmiste et de gobi selon le captureur en zone de forêt.

une densité simulidienne maximale de six piqûres par homme et par jour.

La comparaison deux à deux du nombre de piqûres par homme et par jour, obtenus lors de nos expérimentations en savane (Coco : $2,710^{-6}<\mathrm{p}<0,90$; palmiste : $3,610^{-6}<\mathrm{p}<0,772$; gobi : $10^{-6}<\mathrm{p}<0,277$ ) et en forêt (coco : 0,0105<p $<0,883$; palmiste : $210^{-7}<\mathrm{p}<0,09$ ; gobi : $\left.10^{-6}<\mathrm{p}<0,909\right)$ indiquent qu'un même répulsif peut conférer une qualité de protection différente selon le captureur. Toutefois, au regard du nombre de piqûres par homme et par jour, nos résultats permettent de retenir que quel que soit le répulsif appliqué en savane ou en forêt il protège tous les captureurs contre la nuisance simulidenne. Certes, le seuil de nuisance de 30 piqûres par homme et par jour a été atteint ou dépassé chez le captureur $\mathrm{n}^{\circ} 1$ en forêt (palmiste : 30 piqûres/homme/jour; gobi : 49), mais, pendant la même période, le témoin enregistrait 533 piqûres par jour. Par ailleurs, l'assèchement rapide des produits appliqués sur le captureur $n^{\circ} 1$ pourrait expliquer cette différence par rapport aux autres captureurs

Il est à noter que cette constatation rappelle celle faite par Pitroipa (2000) à propos de la quantité de répulsif à appliquer selon la constitution physique (taille, robus- tesse) du captureur. Malgré cette réserve, nos travaux permettent de retenir que, pour une zone donnée, les répulsifs testés confèrent une protection similaire à tous les captureurs. Certes, le nombre de piqûres par homme et par jour peut varier d'un captureur à l'autre, mais cette différence s'est traduite dans nos résultats par une densité simulidienne inférieure au seuil de nuisance.

Les expérimentations effectuées en savane et en forêt permettent de retenir que les formulations lotions et crèmes des répulsifs testés (coco, palmiste, gobi) s'avèrent dotées d'un pouvoir répulsif capable de protéger une personne contre la nuisance simulidienne. En effet, lorsque nous comparons l'efficacité de ces formulations à celle du DEET, qui est actuellement le produit de synthèse le plus populaire et le plus utilisé (Carnevale et Mouchet, 1997), il ressort que nos produits présentent une capacité répulsive similaire. En outre, le recours à ces formulations (lotions, crèmes) permet d'éliminer certains inconvénients de l'huile brute (notamment évaporation rapide après application sur la peau, conservation délicate) et surtout ceux du DEET (coût : Hougard et al., 1998 - toxicité : Zadicoff, 1979; Heick et al., 1980 - durée d'action : Spencer et al., 1977; Mehr et al., 1985; Rutledge et al., 1996). 


\section{CONCLUSION}

M algré les variations importantes dans le nombre de simulies capturées en fonction du captureur choisi, nos résultats montrent que quels que soient le répulsif appliqué et l'espèce simulidienne, une protection similaire est conférée à tous les captureurs. Ils indiquent également que le recours à des substances naturelles locales, utilisées comme répulsifs en application dermique, est l'une des meilleures méthodes de protection personnelle contre les simulies. Toutefois, il est indispensable de poursuivre les travaux pour aboutir à la mise au point d'un répulsif efficace, acceptable par les populations rurales, d'utilisation facile, d'un coût très bas et surtout inoffensif pour l'homme.

\section{RÉFÉRENCES}

ADossi M. Contribution à la lutte contre l'onchocercose dans le contexte de l'après OCP. II - Évaluation des répulsifs contre Simulium damnosum s. 1. Theobald 1903 (Diptera : Simuliidae). Mémoire de DEA, Université de Côte d'Ivoire (CEMV de Bouaké), 1994, n 43, 27-48.

ANonyme. Protection contre les piqûres de simulies (additif au document OCP/EAP/Briefing). Comité consultatif d'experts : $18^{\text {ème }}$ session, 2-6 juin 1997; Session débriefing, 31 mai 1997.

Bounkoungou M. Ivermectine (Mectizan ${ }^{\circledR}$, MK 933) et lutte contre l'onchocercose en zone de savane de Côte d'Ivoire : exemple de la région de Fétékro sur le bassin du Nzi. Diplôme d'études approfondies, Université de Bouaké, 1999, 55 pp.

Carnevale P. \& Mouchet J. La protection individuelle contre les insectes vecteurs. Médecine Tropicale, 1997, (57 bis), 505-510.

DukE B.O.L. Studies of factors influencing the transmission of Onchocerciasis. IV. The biting cycles, infective biting density and transmission potential of forest Simulium damnosum. Annals of Tropical Medecine and Parasitology, 1968, 62 (1), 95-106.

Garms R. \& Zilimann U. Morphological identification of Simulium sanctipauli and Simulium yahense in Liberia and comparaison of results with these of enzyme electrophoresis. Tropenmedizin und Parasitologie 1984, 35, 217-220.

Heick H.M., Shipman R.T., Norman M.G. \& James W. Reye Like syndrome associated with use of insect repellent in a presumed heterozigote for ornithine carbamyl transferase deficiency. Journal of Pediatry, 1980, 97, 471-473.

Hougard J.M., Agoua H., Yameogo L., Akpoboua K.L.B., SEKETELI A. \& DADZIE K.Y. La lutte contre la nuisance simulidienne : quels choix après l'onchocercose? Forum Mondial de la Santé, 1998, 19, 185-288.

Kurtak D.C., Raybould J.H. \& Vajime C. Wing tuff colors in the progeny of single individuals of Simulium damnosum (Enderlein). Transactions of the Royal Society of Tropical Medecine and Hygiene, 1981, 75, 126 pp.

Le Berre R. Contribution à l'étude biologique et écologique de Simulium damnosum Theobald 1903 (Diptera : Simuliidae). Mémoire ORSTOM, 1966, 17, 204 pp.
LEwIS D.J. Simulium damnosum and its relation to onchocerciasis in the anglo-egyptian Sudan. Bulletin of Entomological Research, 1953, 43 (4), 597-644.

Mehr Z.A., Rutledge L.C., Morales E.L. et al. Laboratory évaluation of controlled release insect repellent formulations. Journal of American Mosquitoes Control Association, 1985, 1, 143-147.

NoAmesi G. Preliminary report on a new repellent "Simno" against blackflies and other biting insects. Ghana Medecine Journal, 1970, 9, 39-43.

Opuku A.K., Rayboulld J.N. \& Kessie D.K. Preliminary field evaluation of the repellent "Simno" against the black fly Simulium damnosum s. 1. a biting midge and mosquitoes. Insect Science and its application, 1985, 7, 31-35.

Philippon B. Étude de la transmission d'Onchocerca volvulus (Leuckart, 1893) (Nematoda, Onchocercidae) par Simulium damnosum Theobald 1903 (Diptera, Simuliidae) en Afrique tropicale. Thèse de Doctorat d'État ès Sciences Naturelles. Paris, 1977, 308 pp.

Pitroipa X. Évaluation de méthodes traditionnelles de protection contre la nuisance due à Simulium damnosum s. 1., vecteur de la cécité des rivières en Afrique de l'Ouest. Mémoire de DEA-Université de Côte d'Ivoire (CEMV de Bouaké), 2000, n 004, 55 pp.

Quilevere D. Contribution à l'étude des caractéristiques taxonomiques bio-écologiques et vectrices des membres du complexe Simulium damnosum présents en Côte d'Ivoire. Travaux et documents de l'ORSTOM, 1979, 109 (1), 304 pp.

Renz H. \& Enyong P. The efficacy of appropriate cloting and DEET Simulium repellent as an individual protection against the transmission of onchocerciasis. Tropical Medicine and Parasitology, 1987, 38, 267-271.

Rutledge L.C., GuPtA R.K. \& Meur Z.A. Evaluation of controlled release mosquito repellent formulation. Journal of American Mosquitoes Control Association, 1996, 12, 39-44.

SснміDт M.L. Relative effectiveness of repellents againt Simulium damnosum (Diptera : Simuliidae) and Glossina morsitans (Diptera : Glossinidae) in Ethiopia. Journal of Medical Entomology, 1977, 14, 276-278.

Schreck C.E., Posey K. \& SMith D. Repellency of selected compound against blackflies (Diptera : Simuliidae). Journal of Medical Entomology, 1979, 15 (5-6), 526-528.

Spencer T.S., Hill J.A., Akers W.A. \& Bjorkland G. Studies of repellent formulations with $\mathrm{N}$ diethyl-n-toluamide. Annual Confernce California Mosquitoes Vector Control Association, 1977, 45, 121-123.

Sylla M.L. Évaluation de l'efficacité de différentes formulations de répulsifs à base d'huiles végétales contre les piqûres de Simulium damnosum s. I. en Côte d'Ivoire. Mémoire de DEA, Université de Bouaké (CEMV), 2001, n 05, 66 pp.

Wilson M.D., POST R.J. \& GOMUlski L.M. Multivariate morphotaxonomie in the identification of adult females of the Simulium damnosum Theobal complex (Diptera : Simuliidae) in the Onchhocerciasis Control Programme area of West Africa. Annals of Tropical Medecine and Parasitology, 1993, 87, 65-82.

ZaDicoff C.M. Toxic encephalopathy associated with use of insect repellent. Pédiatry, 1979, 95, 140-142.

Reçu le $1^{\text {er }}$ mars 2002 Accepté le 5 juillet 2002 